\title{
Performance Evaluation Method of Organic Photovoltaics under Indoor Light Condition
}

\section{Hidenori SAITO, ${ }^{a}, c, *$ Daisuke AOKI, ${ }^{a}$ Shinichi MAGAINO, ${ }^{a}$ Katsuhiko TAKAGI, ${ }^{b}$ and Shuzi HAYASEc}

\author{
a Kanagawa Institute of Industrial Scientific and Technology (KISTEC), \\ 3-2-1 Sakado, Takatsu, Kawasaki, Kanagawa 213-0012, Japan \\ b Research Association Technology Innovation of Organic Photovoltaics (RATO), \\ 4-6-1 Komaba, Meguro, Tokyo 153-8904, Japan \\ c Kyushu Institute of Technology, 2-4 Hibikino, Wakamatsu, Kitakyushu, Fukuoka 808-0196, Japan \\ * Corresponding author: saito@newkast.or.jp
}

\begin{abstract}
Organic photovoltaics are expected to be suitable for indoor use because they have a relatively high conversion efficiency even at a low irradiance when compared to crystalline silicon photovoltaics. However, the performance measurement procedures of such photovoltaics under indoor light have not been established. In this paper, the illuminance fluctuation of a commercially-available light device originating from an AC power source has been precisely investigated, and the methods to decrease the influence of this fluctuation on the J-V measurements of the photovoltaics were proposed. Furthermore, an LED light irradiation apparatus was developed for more reliable and reproducible J-V measurements of the photovoltaics. This apparatus was found to provide an excellent time stability of illuminance and in-plane uniformity, both of which satisfy the Class A solar simulator permitted by IEC 60904-9. J-V characteristics of the photovoltaics determined by using this apparatus was coincident with that determined by using a commercially-available LED light driven by an AC power source. Finally, it was confirmed that the conversion efficiency of a DSC is greater than that of a Si photovoltaic under the indoor light condition, the illuminance of which is several tens to hundreds Ix.
\end{abstract}

(c) The Electrochemical Society of Japan, All rights reserved.

Keywords : Indoor, Low Illuminance, Organic Photovoltaic, LED

\section{Introduction}

In the past several years, the Kanagawa Institute of Industrial Science and Technology (KISTEC) has been studying evaluation methods of organic photovoltaics since joining the Funding Program of World-Leading Innovative R \& D of Science and Technology (FIRST-PG) started in 2011., ${ }^{1,2}$ It has been clarified that the reproducibility of measurements is improved by careful attention to the positioning of a sample cell, light shielding mask for area regulation, and prevention of reflections from the surroundings based on previous studies. The performance evaluation method of photovoltaics is based on the evaluation method of a Si photovoltaic under STC $\left(1 \mathrm{kWm}^{-2}, 25^{\circ} \mathrm{C}\right)$, and standardized in the IEC 60904 series. However, emerging photovoltaics, for example, a dyesensitized solar cell (DSC), an organic thin-film photovoltaic (OPV), and a perovskite solar cell (PSC) need additional measurement methods because such photovoltaics have the advantage in the case of uses different from that of Si photovoltaics. ${ }^{3-6}$ The performance measurement procedures of such photovoltaics have not been established as an international standard by the IEC. Therefore, characterization of organic photovoltaics tends to be carried out according to the evaluation methods of each institute based on their own methods at the present time. ${ }^{7-16}$

Independently, in the past years, in relation to environmental problems, a suppression of the increasing greenhouse gases has been now undertaken on a worldwide scale. In parallel to this movement, a $25 \%$ decrease in the emitted $\mathrm{CO}_{2}$ gas volume will be scheduled in Japan to be attained by 2030 as compared with that in 2013 . The most striking and popular technology should be the used of solar cells among the renewable energy suppliers. In fact, the generated electricity by solar cells has amounted to over $40 \mathrm{GW}$ in 2016 , exceeding the electricity produced by all the nuclear power plants in Japan. In order to further disseminate solar cells in the future, it is necessary to establish a performance evaluation method suitable for the environment to use. One characteristic of organic photovoltaics is that the power generation efficiency is less likely to deteriorate even at a low illuminance. Hence, they are expected to be appropriate for the use as standalone electric sources by a scattered light reflected from the north-faced walls of buildings or in the woods of mountainside areas. ${ }^{3}$ Additionally, their indoor use under artificial illumination has been envisaged as emergent self-emitting guides is now in progress ${ }^{17}$ for lightings at one's feet and a power source for telecommunication equipment, and human sensors in guest rooms at a hotel. The irradiance of these indoor setups remains at around several tens to hundreds $1 \mathrm{x}$, which can be assumed to be suitable for organic photovoltaics. Indeed, the organic photovoltaics acquired by KISTEC are required to be evaluated at a low irradiance less than the STC.

In addition, the organic photovoltaics are freely adjustable in their cell size, since they can be fabricated by a roll-to-roll technique on a plastic or glass substrate. In this sense, they are applicable in size from square centimeters to mega-solar type panels. Thus, it is probable that the organic photovoltaics can be installed by simply putting in an area without any electric power supply.

In the present paper, special attention to the evaluation of photovoltaics under an indoor light condition is presented. Furthermore, a prototype indoor light irradiation system has been developed using a stable LED light source for indoor performance evaluation of the photovoltaics. 
Commercially-available

LED light source

driven by AC power source

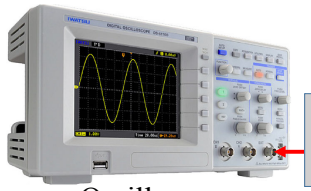

Oscilloscope
Zero shunt ammeter

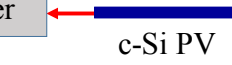

Figure 1. The experimental setup to examine influence of light blinking originating from an $\mathrm{AC}$ power source on the current of a c-Si PV.

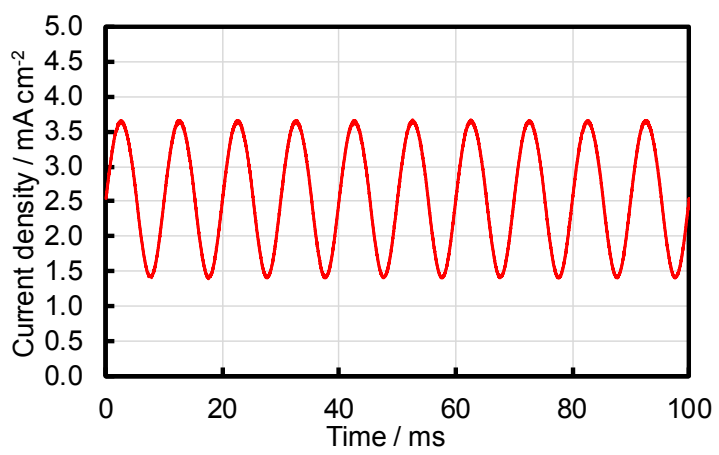

Figure 2. Current density fluctuation of c-Si PV (power generation area: $1.96 \mathrm{~cm}^{2}$ ) caused by light blinking of a lighting apparatus (ALPHAX Co., Ltd. Type AL-50FL).

\section{Evaluation of Photovoltaics under a Commercially-available Lighting Equipment Driven by an AC Power Source}

\subsection{Illuminance measurements}

The performance evaluation of photovoltaics for indoor use is often carried out using a commercially-available lighting equipment driven by an AC power source such as a LED desk lamp. Since the voltage of the $\mathrm{AC}$ power changes at the frequency of $50 \mathrm{or} 60 \mathrm{~Hz}$ as a sinusoidal wave, most of the lighting apparatus is often blinking at the frequency of 100 or $120 \mathrm{~Hz}$ after full wave rectification, which cannot be detected by the human eye.

An experimental setup to examine the influence of light blinking originating from an $\mathrm{AC}$ power source on the current of a crystalline silicon photovoltaic (c-Si PV) is shown in Fig. 1. As shown in Fig. 2, the current density of the c-Si PV periodically fluctuated with the frequency of $100 \mathrm{~Hz}$ synchronizing light blinking of a lighting apparatus (ALPHAX Co., Ltd. Type AL-50FL) originating from a $50 \mathrm{~Hz}$ AC power source. Such a periodic fluctuation of the current is not observed when commercially-available solar simulators are used as lighting equipment because they are driven by a stable DC power supply.

When the illuminance of the lighting equipment is cyclically changing, the measured illuminance depends on the exposure time and number of integrations by an illuminance meter. For this reason, the measurement condition should be properly controlled to obtain reproducible data.

Figure 3 shows the effect of the exposure time on the illuminance measurement results obtained for the lighting apparatus described above. Since the frequency of the power source was $50 \mathrm{~Hz}$, the light was cyclically blinking at the frequency of $100 \mathrm{~Hz}$. In this case, the exposure time should be set to an integral multiple of $10 \mathrm{~ms}$ to avoid scatter in the illuminance measurements results.

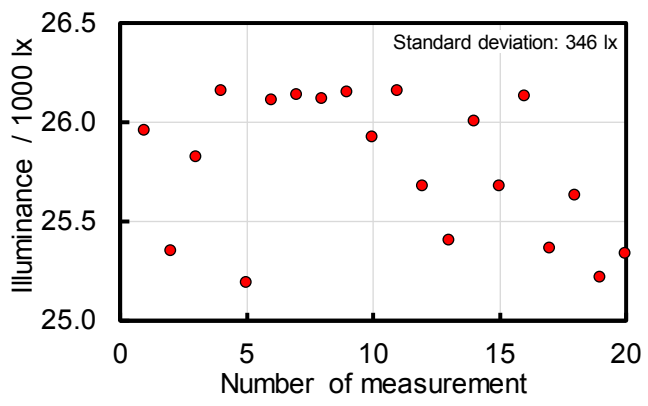

(a)

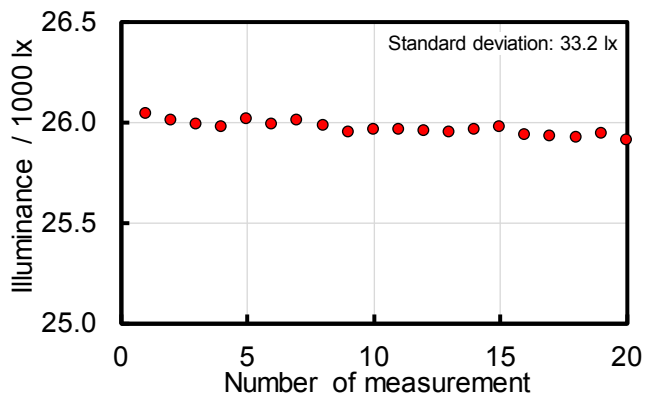

(b)

Figure 3. Effect of the exposure time on illuminance measurement results repeated 20 times determined for a lighting apparatus (ALPHAX Co., Ltd. Type AL-50FL): number of integrations was set to 5; exposure time was set to $12.8 \mathrm{~ms}$ in the automatic mode for (a), and to $10 \mathrm{~ms}$ in the manual mode for (b).

When the illuminance meter employed was set in the automatic mode, the exposure time was automatically set at $12.8 \mathrm{~ms}$. In this case, scatter in the illuminance measurement results was quite high since the exposure time was not an integral multiple of $10 \mathrm{~ms}$ as shown in Fig. 3(a). On the other hand, the result obtained when the exposure time was manually set to $10 \mathrm{~ms}$ shows much less scatter as shown in Fig. 3(b). However, the result still had some scatter probably because the number of integrations was as low as 5 . These results show the measured illuminance value significantly varies depending on the measurement conditions even though the quantity of light is exactly the same.

In general, most illuminance meters can be switched between the automatic mode for automatically adjusting the exposure time and the manual mode for manually setting the measurement conditions. When the illuminance is measured under a commercially-available light source directly driven by the AC power source, the manual mode should be always selected and set the exposure time to be an integral multiple of double the AC power cycle period.

The measurement also became unstable when the exposure time was too long. When the exposure time is too long, the spectrum overshoots the dynamic range, so the shape of the spectrum significantly collapses (Fig. S1 in Supporting Information (SI)). The results show that the exposure time should be set to the optimum value within the dynamic range of the employed illuminance meter and be an integral multiple of double the AC cycle period. It is important to repeat the preliminary experiments until the optimum measurement condition is determined.

Figure 4(a) shows the scatter in the illuminance measurement results obtained for the various number of integrations with the fixed exposure time to $10 \mathrm{~ms}$. It is obvious that the scatter is high when the number of integrations is low. Figure 4(b) shows the relationship between the instability of the measured illuminance and the number of integrations. The instability was calculated from the maximum illuminance $\left(E_{\max }\right)$ and minimum illuminance $\left(E_{\min }\right)$ as follows: 


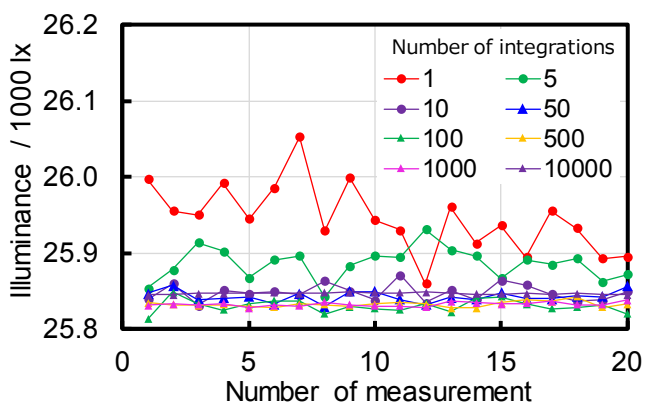

(a)

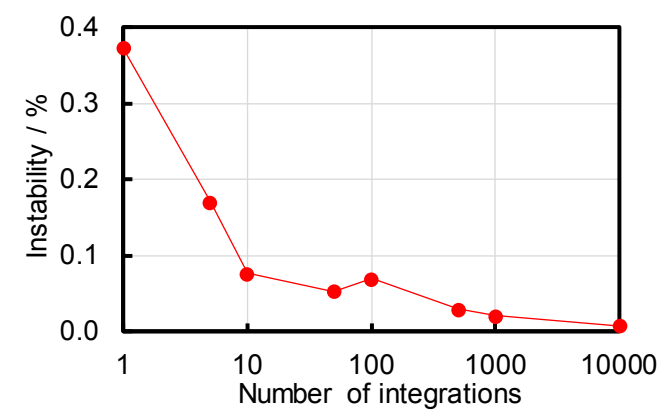

(b)

Figure 4. Illuminance measurement results for a lighting apparatus (ALPHAX Co., Ltd. Type AL-50FL) when the number of integrations is changed with the fixed exposure time of $10 \mathrm{~ms}$ : (a) scatter of illuminance measurement results for the various number of integrations; (b) relation between the instability of measured illuminance and the number of integrations.

$$
\text { Instability }=\frac{E_{\max }-E_{\min }}{E_{\max }+E_{\min }} \times 100 .
$$

In this experiment, the instability was measured by setting the number of integrations to 10000 times, and it was found that 500 integrations, which needs about a 5 -s duration, might be practically sufficient to obtain reliable data.

\section{$2.2 \mathrm{~J}-\mathrm{V}$ measurements}

The proper setting of the integration time is important not only for the illuminance measurement, but also for the J-V measurement of the photovoltaics. Effect of the integration time on the J-V curve of a c-Si PV measured by a LED bulb (SHARP corp. Type DLLA8AN) and a source meter (ADC Corp. type 6246) is shown in Fig. 5. The measured current was influenced by the AC power source frequency when the integration time is short, so it is easy to notice the mistake, and conversely, as the integration time is lengthened, it converges to the central values.

Figure 6 shows the J-V curves obtained for the same photovoltaics, but using a different source meter manufactured by a different company. Since this source meter was designed only for stable light from a solar simulator or sunlight, the measurement conditions cannot be precisely controlled. Therefore, when a commercially-available lighting apparatus driven directly by an AC power source is used, the reproducibility of the $\mathrm{J}-\mathrm{V}$ curve measurement is quite poor as shown in Fig. 6. Since the illuminance of this experiment was the same as that of the experiment shown in Fig. 5, the J-V curves passed somewhere in the range shown in Fig. 5.

In addition, the current densities of the 1st result in Fig. 6 are relatively high. The higher current densities are thought to be measured by sampling at the time when the illuminance was higher than the average value. This result demonstrates that careful

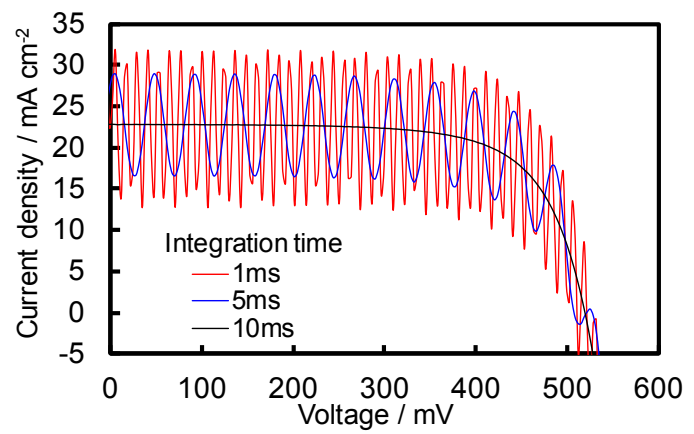

Figure 5. Effect of the integration time on the J-V curve of a c-Si (power generation area: $1.96 \mathrm{~cm}^{2}$ ) PV measured by using a LED bulb (SHARP corp. Type DL-LA8AN) and the type 6246 source meter manufactured by the ADC Corporation.

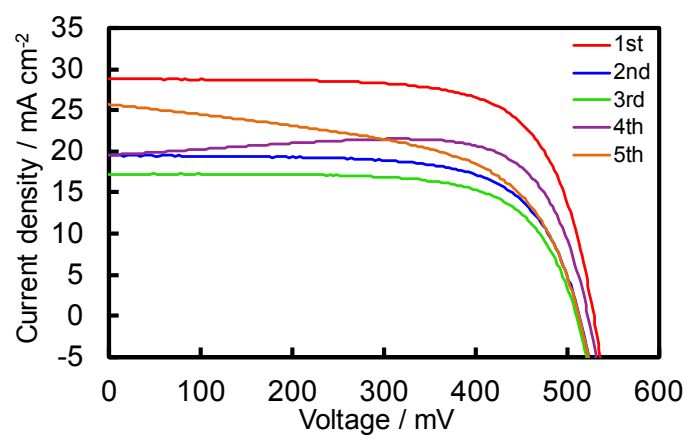

Figure 6. Variation in the $\mathrm{J}-\mathrm{V}$ curve determined for a c-Si PV (power generation area: $1.96 \mathrm{~cm}^{2}$ ) by using a LED bulb (SHARP corp. Type DL-LA8AN) and a source meter designed only for stable light.

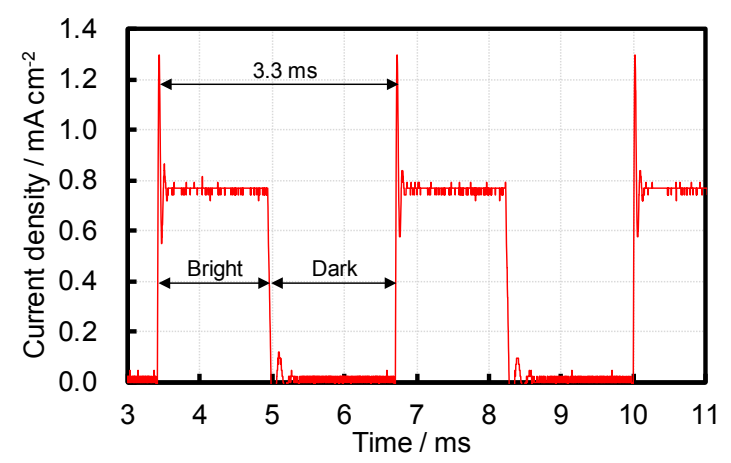

Figure 7. Example of the current pattern of a c-Si PV determined under a LED lighting apparatus that controls illuminance by on-off time with pulse waves: dimming level was about $50 \%$ in this case.

attention must be paid because an apparently high performance can be obtained depending on the source meter.

\section{$2.3 \mathrm{~J}-\mathrm{V}$ measurements under the LED light equipped with illuminance control function}

In recent years, there are an increasing LED lighting apparatus that controls illuminance by on-off time with pulse waves. Figure 7 shows an example of the current pattern of a c-Si PV under such a lighting apparatus. Brightness of the LED light source (MatsukiGiken Co., Ltd. MDL-LE841 type desk light) used in this experiment was changed by controlling the time of turning on and 


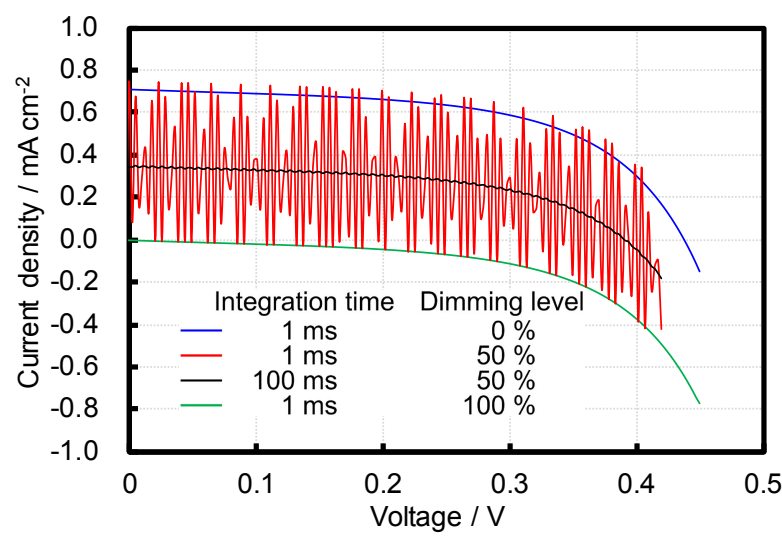

Figure 8. Change in the J-V curve of c-Si PV (power generation area: $2.6 \mathrm{~cm}^{2}$ ) with the dimming level and the integration time.

off (Fig. S2 in SI). Lighting apparatuses except for this type have no time to completely turn off for dimming.

Figure 8 shows change in the J-V curve of a c-Si PV with the dimming level and the integration time. As the lighting and extinguishing were repeated, the current oscillated between maximum illuminance and dark when the integration time was $1 \mathrm{~ms}$ in the similar manner to the J-V curve shown in Fig. 5. The oscillation decreased with increasing the integration time, but it could not be completely removed even when the integration time was increased to $100 \mathrm{~ms}$ (Fig. S3 in SI).

It is now in progress in our hands to develop a modified evaluation method to get the more accurate physical data for the photovoltaic performance.

\section{Development and Performance of an LED Light Irradiation Apparatus}

\subsection{Development of an LED light irradiation apparatus}

As mentioned in the previous section, there are many variables to be optimized for evaluation of photovoltaics under low illumination assuming indoor use. In order to optimize organic photovoltaics, it is also necessary to establish evaluation methods adequate to characterize organic photovoltaics, for example, high performance under low illumination.

In order to evaluate the power generation characteristics of photovoltaics due to incident light by excluding the illuminance fluctuation caused by the AC power source, we postulated that a dedicated light source for indoor light was necessary. Thus, a stable light irradiation apparatus with a low illuminance using an LED as the light source has been developed and the performance of this setup was evaluated. The reason why the LED was employed as the light source is that it is becoming the main indoor light source replacing the fluorescent light in Japan.

The LED generates light only in the visible region, and there are models that suppress power while ensuring illuminance by pulse lighting, which is different from the conventional artificial light sources such as incandescent bulbs and fluorescent lamps. Initially, the use of a commercially-available LED bulb was considered, but an LED light source was finally designed by our group with the LED element having the feature of being driven by a stable DC power supply, considering the unknown nature of the incorporated LED element and the spectrum changes due to the specification change. The selected LED element was the type 757 which is widely used for lighting produced by the Nichia Corporation.

The LED light irradiation apparatus, was made by the Cell System Co., Ltd., according to our design. Five LED elements were positioned at four top points on an aluminum plane $(226 \mathrm{~mm} \times$
$226 \mathrm{~mm}$ square) with a cross point of the diagonal lines as shown in Figs. 9(a) and (b). Upon illumination of this LED device, the stabilized DC power supply was sufficiently separated so as not to increase the temperature of the device, as depicted in Fig. 9(c). The stabilized DC power supply controlled by a PC supplied the constant current without blinking as shown in Fig. 9(d), then irradiance of the LED light irradiation apparatus, was kept constant for a long time.

A dark box for the measurements having the framework shown in Fig. 10 was also fabricated. Illuminance can be changed not only by the current control using the stabilized DC power supply, but also by changing the height of the plate equipped with the light source. Since the height of the light source can be continuously changed over the range of $1800 \mathrm{~mm}$ by a belt moving, it is possible to set an arbitrary illuminance.

Additionally, a slide rail mechanism is equipped at the bottom of the dark box in order to measure the illuminance (or irradiance) and characteristics of the photovoltaics at almost the same vertical position (Fig. S4 in SI). By using this mechanism, a spectroradiometer and a photovoltaic can be interchanged at almost the same position in one operation. Many of the test photovoltaics currently in use have a small area of $10 \mathrm{~mm}$ square $\left(1 \mathrm{~cm}^{2}\right)$ or less. In the case of such small photovoltaics, the performance tends to significantly fluctuate even with a slight positional deviation. The reproducibility of the positioning might be greatly improved by using this mechanism. A small MS-720 spectroradiometer manufactured by EKO instruments is used in combination with this dark box. Since this spectroradiometer is able to perform automatic interval measurements in addition to remote operation, it is considered to be effective for continuous measurement over a long period of time. This LED light irradiation apparatus, has already been filed as a utility model. In addition, which named "Iris-S T2500".

\subsection{Performance of the LED light irradiation apparatus}

\subsubsection{Time stability of illuminance}

Figures 11(a) and (b) show the time variation of the spectrum, illuminance and temperature determined for Iris-S T2500. This apparatus resulted in the successful improvement of the electric power stability and the heat dissipation ability, and thus the irradiation light spectrum and intensity almost remain constant during the working time of 8 hours. On the contrary, the spectrum, illuminance and temperature of the commercially-available LED bulb (TOSHIBA type LDA6N-H) rapidly changed in about 15 minutes as shown in Figs. 11(c) and (d). Generally, commerciallyavailable LED bulbs increase in temperature by emitting heat. This change in the temperature is assumed to be due to the electric power supply and LED elements. Therefore, the almost constant temperature of Iris-S is believed to be caused by the LED elements that separately from the electric power part as well as by the aluminum plate used as the heat dissipation material for the ceiling plate on which the LED elements are attached.

The time variation rate of the irradiance of this instrument were estimated to be $0.15 \%$ after a 500 -min irradiation from the initial. These variations are satisfactory, considering the time within those of the short $(<0.5 \%)$ and long term instabilities of irradiance $(<2 \%)$ of the Class A solar simulator permitted by IEC 60904-9. ${ }^{18}$ While a commercially-available LED bulb is usually composed of an electric power unit together with the emitting LED elements, the evolved heat can accumulate in the LED bulb, inducing a decrease in the irradiance with an increase in the lightening time, eventually attaining a decrease of $15 \%$ of the initial illuminance. That is, the time variation of the irradiance of the LED bulb resulted in $8.23 \%$ after $500 \mathrm{~min}$, and $0.34 \%$ from $60 \mathrm{~min}$ to $500 \mathrm{~min}$. The commercial LED bulbs are usually unstable, since they are not used for the purpose as an irradiance calibration light. Nevertheless, surprisingly, only few companies usually carry out a performance analysis of the emerging photovoltaics using an LED bulb as the irradiance source, 


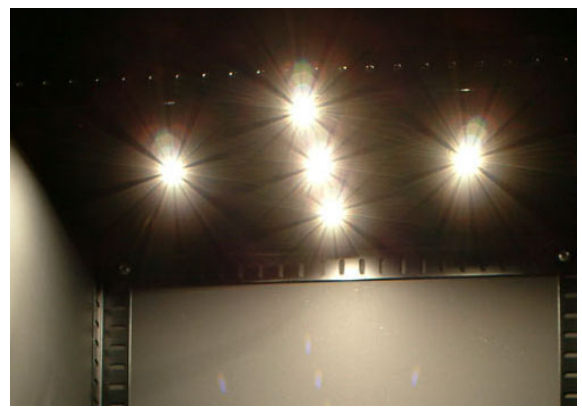

(a)

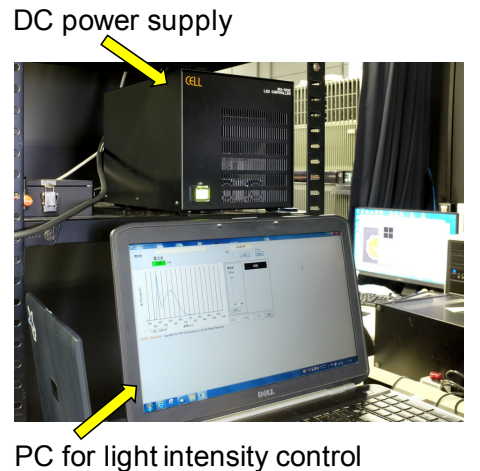

(c)

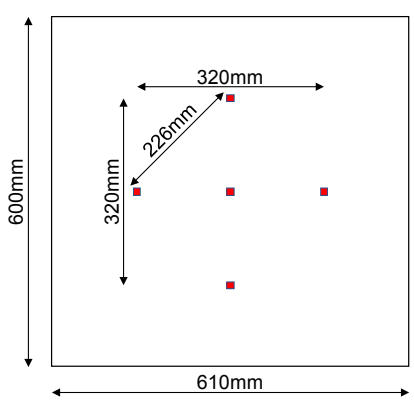

(b)

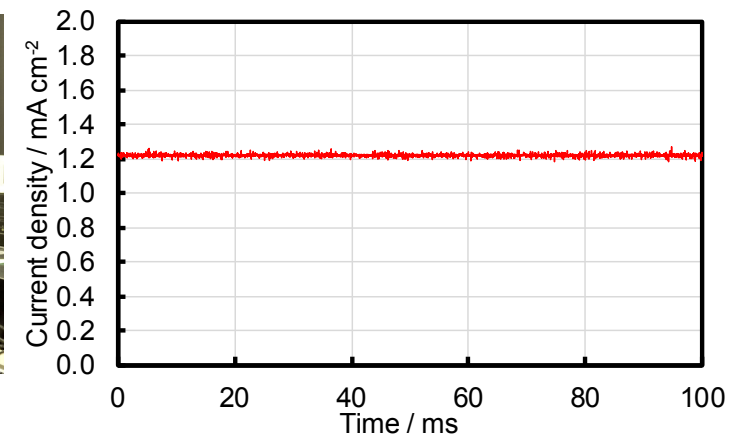

(d)

Figure 9. Fundamental setup of the LED light irradiation apparatus: (a) five LED elements on the aluminum plate; (b) schematic diagram of five LED element arrangement; (c) DC power source and control PC isolated from LED elements; (d) current density measured from c-Si PV (power generation area: $4 \mathrm{~cm}^{2}$ ) without blinking supplied by DC power source.

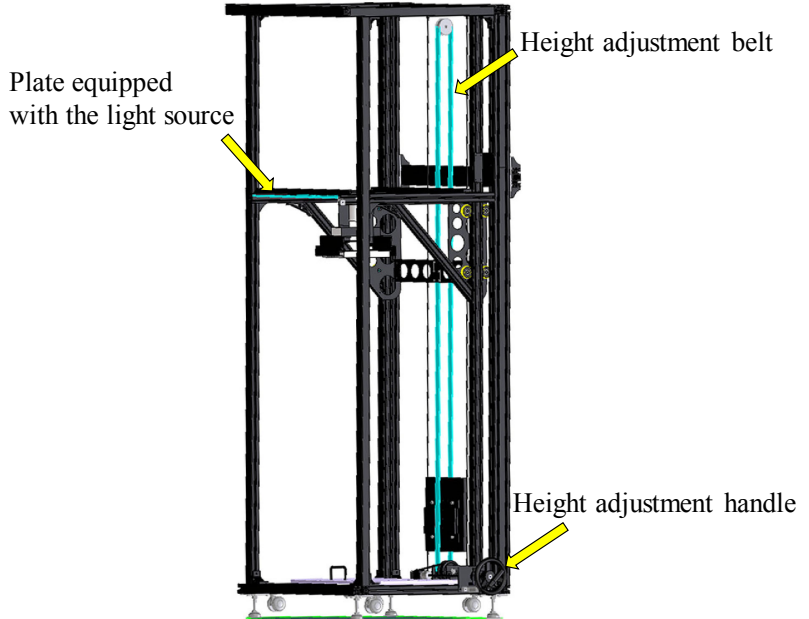

Figure 10. 3D-CAD image of the dark box framework fabricated.

based on the questions from the companies in these photovoltaics markets. It is concluded that in order to carry out a highly and reproducible performance, it should be clearly pointed out that the LED light irradiation apparatus developed in this paper should be used and not the usual LED bulb light source directly driven by an AC power source.

\subsubsection{Uniformity of illuminance distribution}

Figure 12 shows the in-plane illuminance distribution measured by the illuminance distribution measuring device for a solar simulator manufactured by the FA Systems Corporation. The permission value of a solar simulator of non-uniform irradiance on the test plane defines $2 \%$ for Class A, $5 \%$ for Class B, and $10 \%$ for Class C in IEC 60904-9. ${ }^{18}$ In the case of the present LED light irradiation apparatus, the non-uniformity of irradiance on the test plane was estimated to be $1.34 \%, 0.62 \%, 1.05 \%, 1.63 \%$ for the illuminance of 50, 200, 500, and $1000 \mathrm{~lx}$, respectively, all of which are satisfied for Class A.

\subsection{Applicability of the LED light irradiation apparatus to the $\mathrm{J}-\mathrm{V}$ analysis of the photovoltaics operating in an actual environment}

It has been evident by the above-mentioned studies for the validity of the $\mathrm{J}-\mathrm{V}$ analysis of the photovoltaics that the measurements of illuminance and $\mathrm{J}-\mathrm{V}$ characteristics should be performed by setting the integration time and exposure time to be integer multiple of the blinking cycle and long enough under the blinking light directly driven by an $\mathrm{AC}$ power source. In this sense, it was examined in this paper whether $\mathrm{J}-\mathrm{V}$ characteristics determined under the blinking light is coincident with that determined under the light driven by the stabilized DC power supply (Iris-S) developed in this paper when the irradiance is set same to each other.

As a first step, the illuminance and the J-V curves were measured under the commercially available LED blinking light (AL-50FL, ALPHAX Co., Ltd.) directly driven by an AC electric power set illuminance at ca. $200 \mathrm{~lx}$ (Irradiance $=0.058 / \mathrm{mWm}^{-2}$ ). And then by using Iris-S, whose illuminance was accurately adjusted to be $0.058 / \mathrm{mWm}^{-2}$, both the illuminance and the $\mathrm{J}-\mathrm{V}$ measurements were conducted. By using a similar procedure, the $\mathrm{J}-\mathrm{V}$ analysis of $\mathrm{c}-\mathrm{Si} \mathrm{PV}$ (power generation area: $1.93 \mathrm{~cm}^{2}$ ) and DSC (power generation area: $0.196 \mathrm{~cm}^{2}$ ) solar cells were done for comparison of these different kinds of their performance. Similar measurements of these two kinds of the photovoltaics were also examined at ca. $1000 \mathrm{~lx}\left(0.298 / \mathrm{mWm}^{-2}\right)$.

Spectral shapes of AL-50FL and Iris-S are similar but different from each other (Fig. S5 and S6 in SI). However, since the irradiance was almost the same, the power generation behavior was able to obtain close values as shown in Fig. 13. The short circuit current density $\left(\mathrm{J}_{\mathrm{SC}}\right)$, the open circuit voltage $\left(\mathrm{V}_{\mathrm{OC}}\right)$ and the 
Electrochemistry, 86(6), 363-370 (2018)

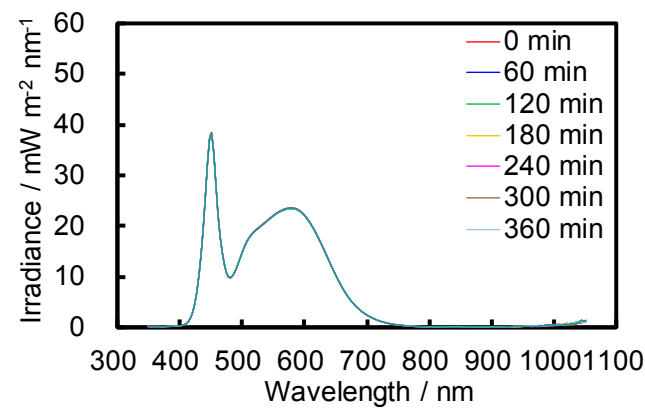

(a)

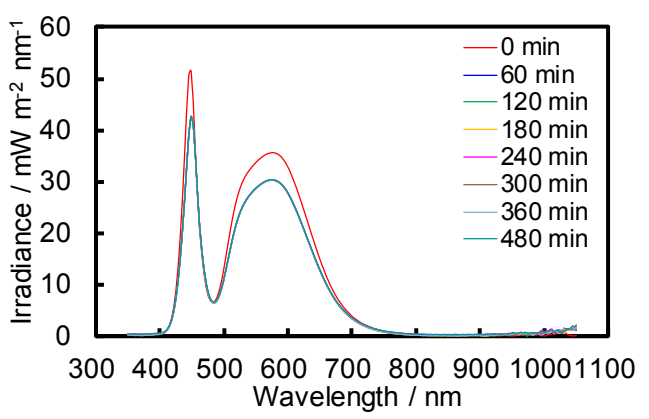

(c)

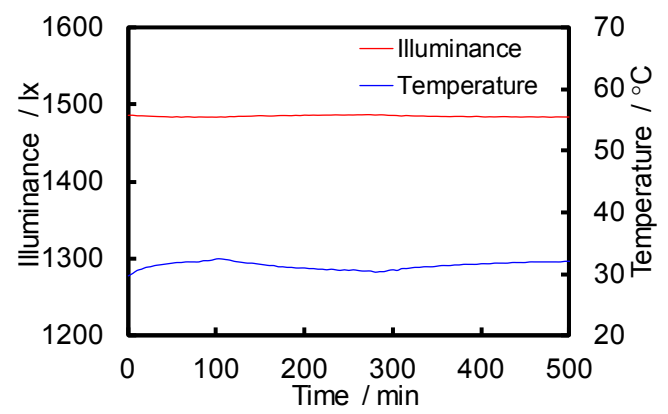

(b)

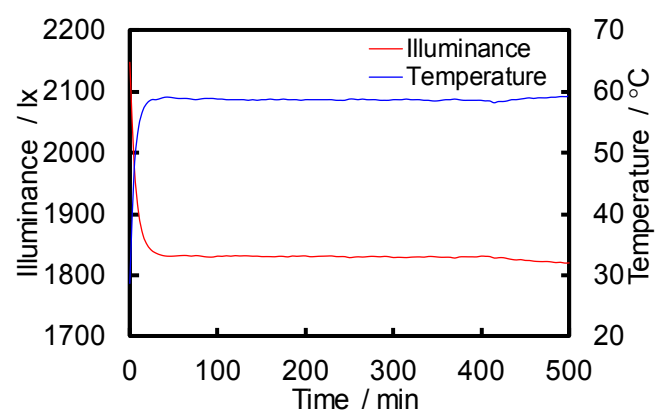

(d)

Figure 11. Time variation of spectrum, illuminance and temperature: (a) spectrum of the LED light irradiation apparatus (Iris-S T2500); (b) illuminance and temperature of Iris-S T2500; (c) spectrum of a commercially-available LED bulb (TOSHIBA type LDA6N-H); (d) illuminance and temperature of LDA6N-H.

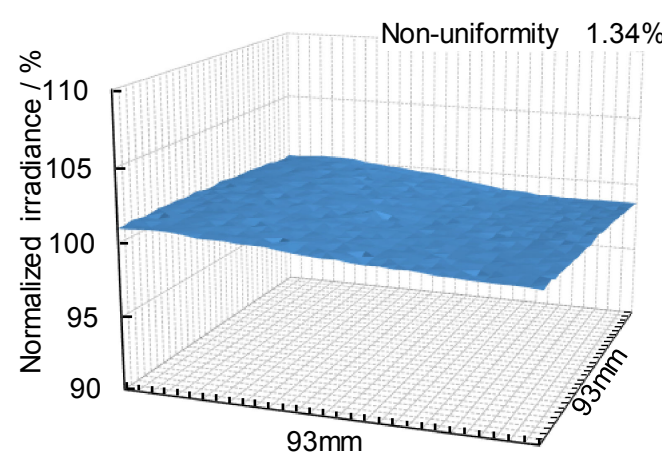

(a)

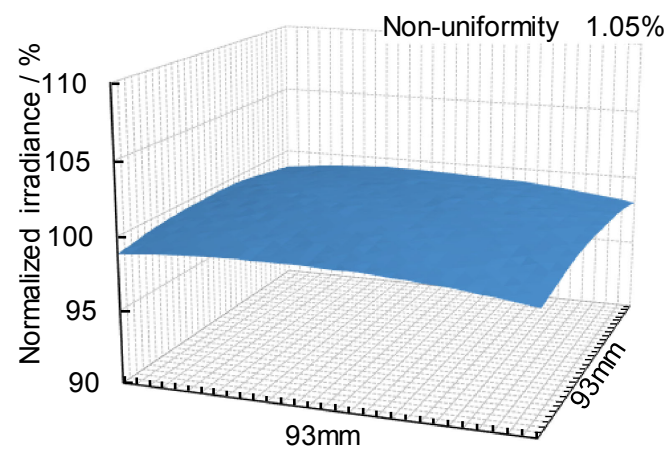

(c)

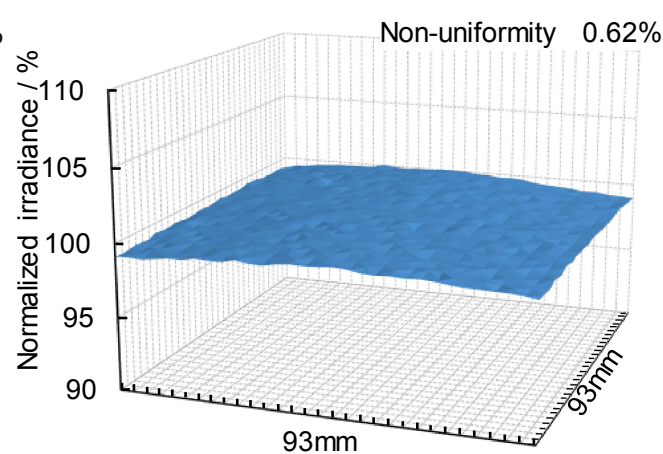

(b)

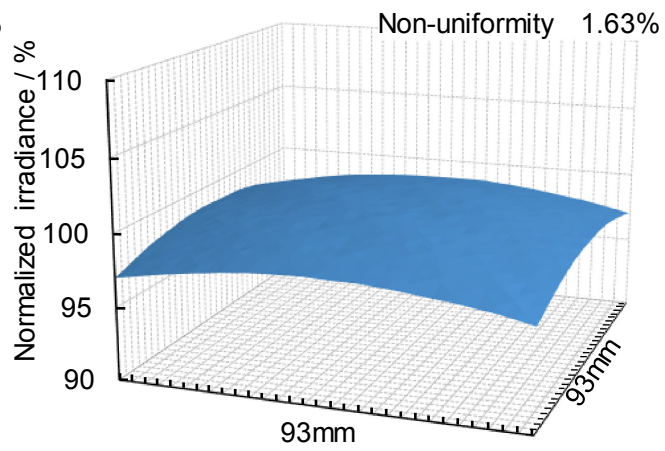

(d)

Figure 12. In-plane uniformity measured for Iris-S T2500: (a) 501x; (b) $2001 x$; (c) $5001 x$; (d) $10001 x$.

maximum power $\left(\mathrm{P}_{\max }\right)$ were shown in Tables $\mathrm{S} 1$ to $\mathrm{S} 4$ in $\mathrm{SI}$. Especially, when the irradiance was high $\left(0.298 \mathrm{mWm}^{-2}\right)$, the difference in $\mathrm{P}_{\max }$ between Iris-S and AL-50FL could be suppressed to $1 \%$ or less. The difference is assumed to be greater at a low irradiance $\left(0.058 \mathrm{mWcm}^{-2}\right)$ due to lower accuracy of the positioning of the photovoltaics samples caused by illuminance inhomogeneity on the sample holder. When the performance of some commercial photovoltaics with a much larger cell size will be necessary to be evaluated in the future, it will be a very important problem to accurately estimate the irradiation intensity on the photovoltaic 


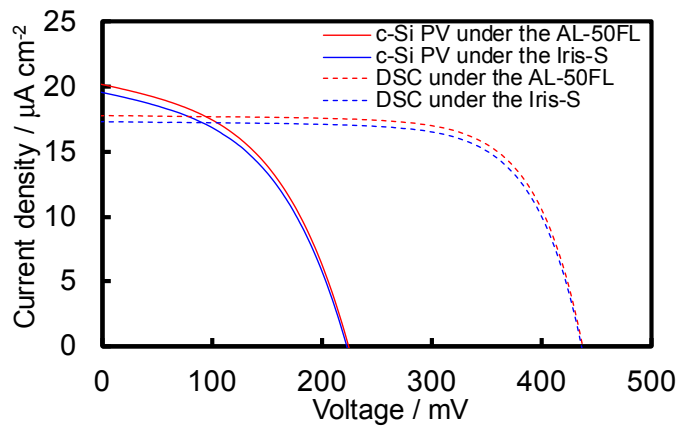

(a) Results at irradiance of about $0.058 / \mathrm{mWm}^{-2}$.

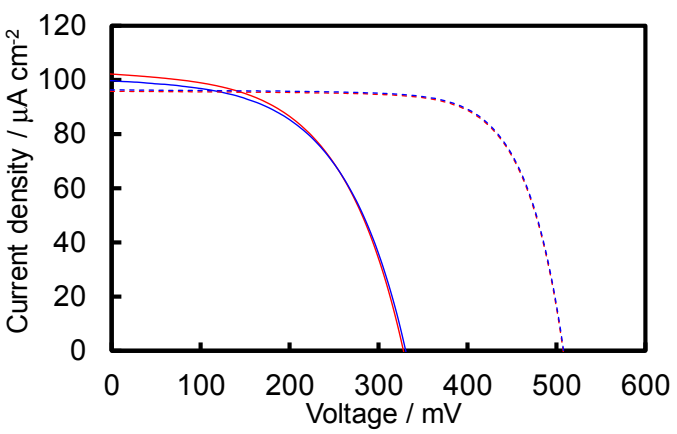

(b) Results at irradiance of about $0.298 / \mathrm{mWm}^{-2}$.

Figure 13. Comparison of J-V measurement results using the AL50FL and Iris-S. Solid lines and dotted lines represents c-Si PV and DSC, respectively. (a) Results at irradiance of about $0.058 / \mathrm{mWm}^{-2}$. (b) Results at irradiance of about $0.298 / \mathrm{mWm}^{-2}$.

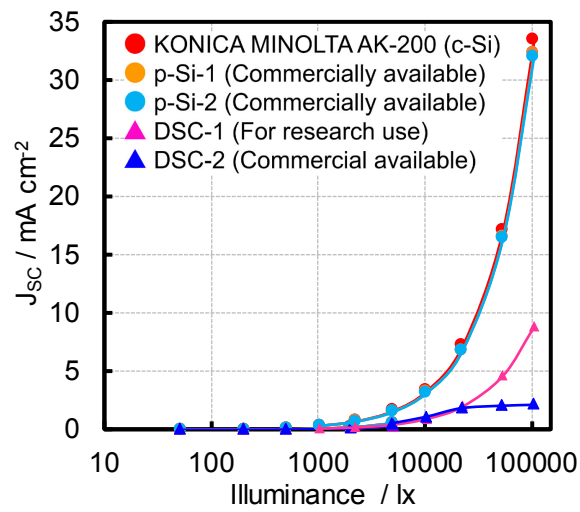

(a)

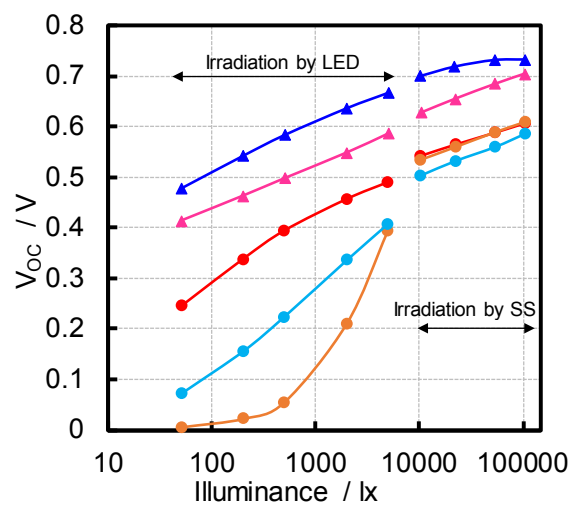

(b) surface receiving incident light, considering their illuminance distribution, the cell size, and the fixed position. In this regard, we have a plan to continue further investigations including the developments of the design of measurement auxiliaries.

According to the present study, it becomes clear that if the illuminance can be measured under appropriate conditions even with AC driven illumination, if the total energy to be irradiated is obtained and the same energy is irradiated under a stable DC driving light source, the reproduced power generation performance is possible. Actual environmental test under the blinking light is also important, but it is very meaningful to estimate a power generation, to characterize the solar cells under the reliable lighting apparatus driven by the stabilized DC power supply.

\subsection{Evaluation of the performance of the photovoltaics}

Recently a number of the organic photovoltaics have been newly developed, after the silicon semiconductor photovoltaics (c-PV) generally prevailed in the world markets. Among these organic PVs, especially DSC is characteristic of its unique performance favorable for dim light illumination, which has often been observed by not a few researchers. That is, we reexamined the influence of illuminance of the incident light in Fig. 14(a)-(d), showing (a) that the $\mathrm{J}_{\mathrm{SC}}$ of both DSC and c-Si PV decrease with decreasing the irradiance. However, either of the $\mathrm{V}_{\mathrm{OC}}$ and the fill factor (FF) of DSC keeps rather high, as shown in (b) and (c). As a result, the power conversion efficiency (PCE) of DSC definitely exceeding over that of c-Si PV at lower irradiance conditions.

On considering the above advantage of the organic PV, especially DSC, over conventional c-Si PV under the low irradiance intensities by artificial lamp like LED or by the sun light incident to the northdirected building walls, the organic PVs will provide a nice usage for the performance suitable for the sensor batteries of IoT devices.

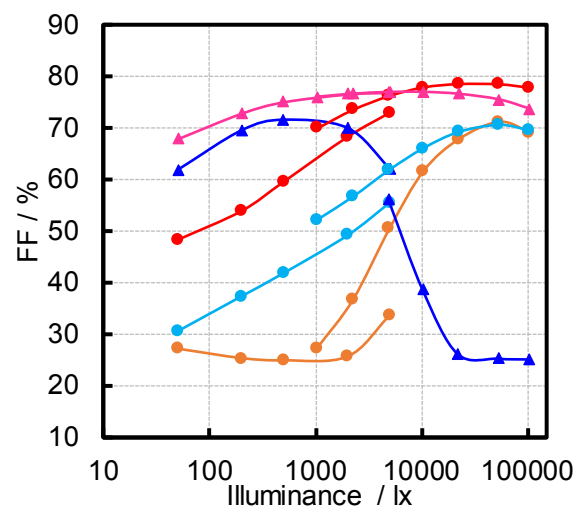

(c)

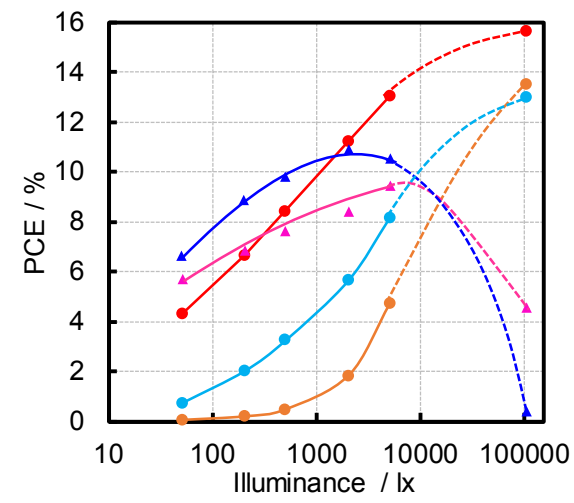

(d)

Figure 14. J-V characteristics of photovoltaics at various irradiance determined by using the LED light irradiating apparatus (Iris-S T2500) developed in this paper: (a) $\mathrm{J}_{\mathrm{SC}}$; (b) $\mathrm{V}_{\mathrm{OC}}$; (c) FF; (d) PCE. A solar simulator was used for the measurements at 100,000 lx. 
Accordingly, it should be meaningful to establish the standard evaluation method of organic PVs including various types like DSC, OPV, PSC, etc. However, since the only organic PV that can be purchased at the moment is DSC, we are mainly considering DSC.

Finally, in the near future we would like to establish the evaluation methodology for the perfect understanding of the newer emerging PVs under a lower illuminance in the indoor/outdoor circumstance, in comparing with DSC and c-Si PVs performance behavior.

\section{Conclusion}

The illuminance fluctuation caused by an $\mathrm{AC}$ power source has been precisely investigated, and the methods to decrease the influence of this fluctuation on the J-V measurements of the photovoltaics were proposed. The LED light irradiation apparatus was developed for more reliable and reproducible J-V measurements of the photovoltaics. In order to eliminate the fluctuation caused by an AC power source, the LED light was driven by a stable DC power supply. In addition, a dark box equipped with this LED light and a mechanism for reproducible positioning of the photovoltaic and spectroradiometer was fabricated. This LED light irradiation apparatus has already been filed as a utility model. The LED light irradiation apparatus developed in this investigation has provided an excellent illuminance stability vs. time and in-plane uniformity, both of which satisfy the Class A solar simulator permitted by IEC 60904-9.

J-V characteristics of the photovoltaics determined by using the LED light irradiation apparatus driven by a DC power supply was coincident with that determined by using a commercially available LED light directly driven by an AC power source when the irradiance is set same to each other.

The J-V curve of a photovoltaic was measured under a dimmable LED light operated by a pulsed current, but a fluctuation originated from a pulsed current could not be completely eliminated even when the integration time was changed. It is now in progress in our hands to develop modified evaluation methods to get the more accurate physical data for the photovoltaic performance.

Finally, it was confirmed that the conversion efficiency of the DSCs is greater than that of a Si photovoltaic under indoor light conditions, the illuminance of which is several tens to hundreds $1 \mathrm{x}$.

\section{Supporting Information}

The Supporting Information is available on the website at DOI: https://doi.org/10.5796/electrochemistry.18-00027.

\section{Acknowledgments}

The authors would like to express their gratitude to Prof. Hiroshi Segawa, The University of Tokyo, Advanced Research Center, Research Center for Advanced Science and Technology.

An acknowledgement is also for the New Energy, Ind. Tech. Development Organization for a Financial Support of Establishment of Highly Reliable Method of Perovskite PV for 2015-2018.

\section{References}

1. D. Aoki, T. Aoki, H. Saito, S. Magaino, and K. Takagi, Electrochemistry, 80, 640 (2012).

2. K. Takagi, S. Magaino, H. Saito, T. Aoki, and D. Aoki, J. Photochem. Photobiol., C, 14, 1 (2013).

3. C. López-López, S. Colodrero, M. E. Calvo, and H. Míguez, Energy Environ. Sci., 6, 1260 (2013).

4. F. De Rossi, T. Pontecorvo, and T. M. Brown, Appl. Energy, 156, 413 (2015).

5. T. M. W. J. Bandara, W. J. M. J. S. R. Jayasundara, H. D. N. S. Fernado, M. A. K. L. Dissanayake, L. A. A. De Silva, I. Albinsson, M. Furlani, and B.-E. Mellander, J. Appl. Electrochem., 45, 289 (2015).

6. L. Cojocaru, S. Uchida, K. Tamaki, P. V. V. Jayaweera, S. Kaneko, J. Nakazaki, T. Kubo, and H. Segawa, Sci. Rep., 7, 11790 (2017).

7. K. Hara, S. Igari, S. Takano, and G. Fujihashi, Electrochemistry, 73, 887 (2005) [in Japanese].

8. F. Cao, G. Oskam, G. J. Meyer, and P. C. Searson, J. Phys. Chem., 100, 17021 (1996).

9. P. M. Sommeling, H. C. Rieffe, J. M. Kroon, J. A. M. van Roosmalen, A. Schönecker, and W. C. Sinke, Proceeding of the $14^{\text {th }}$ European Photovoltaic Solar Energy Conference, p. 1816 (1997).

10. P. M. Sommeling, H. C. Rieffe, J. A. M. van Roosmalen, A. Schönecker, J. M. Kroon, J. A. Wienke, and A. Hinsch, Sol. Energy Mater. Sol. Cells, 62, 399 (2000).

11. T. Trupke and P. Würfel, J. Phys. Chem. B, 104, 11484 (2000).

12. J. Hohl-Ebinger, A. Hinsch, R. Sastrawan, W. Warta, and U. Würfel, Proceeding of the $19^{\text {th }}$ European Photovoltaic Solar Energy Conference, p. 173 (2004).

13. L. J. Diguna, M. Murakami, A. Saito, Y. Kumagai, T. Ishihara, N. Kobayashi, Q. Shen, and T. Toyoda, Jpn. J. Appl. Phys., 45, 5563 (2006).

14. J. Halme, G. Boschloo, A. Hagfeldt, and P. Lund, J. Phys. Chem. C, 112, 5623 (2008).

15. H. Tian, L. Liu, B. Liu, S. Yuan, X. Wang, Y. Wang, T. Yu, and Z. Zou, J. Phys. D: Appl. Phys., 42, 045109 (2009).

16. X.-Z. Guo, Y.-H. Luo, Y.-D. Zhang, X.-C. Huang, D.-M. Li, and Q.-B. Meng, Rev. Sci. Instrum., 81, 103106 (2010).

17. M. Kawaraya, T. Toyoda, S. Doi, D. Kitazawa, H. Saito, H. Segawa, and K. Takagi, Proceedings of International Conference Asia-Pacific Hybrid and Organic Photovoltaics 2018 (AP-HOPV18), poster 141 (2018).

18. IEC 60904-9, Solar simulator performance requirements, Edition 2.0, 2007-10. 